\title{
Anticholinergic and benzodiazepine medication use and risk of incident dementia: a UK cohort study
}

\author{
Carlota M. Grossi ${ }^{1}$, Kathryn Richardson ${ }^{1 *}$ (D) Chris Fox ${ }^{2}$, lan Maidment ${ }^{3}$, Nicholas Steel ${ }^{2}$, Yoon K. Loke ${ }^{2}$, Antony Arthur ${ }^{1}$, \\ Phyo Kyaw Myint ${ }^{4}$, Noll Campbell ${ }^{5}$, Malaz Boustani ${ }^{6}$, Louise Robinson ${ }^{7}$, Carol Brayne ${ }^{8}$, Fiona E. Matthews ${ }^{7}$ and \\ George M. Savva ${ }^{1,9}$
}

\begin{abstract}
Background: Studies suggest that anticholinergic medication or benzodiazepine use could increase dementia risk. We tested this hypothesis using data from a UK cohort study.

Methods: We used data from the baseline (Y0), 2-year (Y2) and 10-year (Y10) waves of the Medical Research Council Cognitive Function and Ageing Study. Participants without dementia at Y2 were included $(n=8216)$. Use of benzodiazepines (including nonbenzodiazepine Z-drugs), anticholinergics with score 3 (ACB3) and anticholinergics with score 1 or 2 (ACB12) according to the Anticholinergic Cognitive Burden scale were coded as ever use (use at Y0 or $Y 2)$, recurrent use (Y0 and $\left.Y_{2}\right)$, new use $\left(Y_{2}\right.$, but not $\left.Y 0\right)$ or discontinued use (Y0, but not $\left.Y_{2}\right)$. The outcome was incident dementia by $\mathrm{Y} 10$. Incidence rate ratios (IRR) were estimated using Poisson regression adjusted for potential confounders. Pre-planned subgroup analyses were conducted by age, sex and Y2 Mini-Mental State Examination (MMSE) score.

Results: Dementia incidence was 9.3\% ( $N=220$ cases) between $\mathrm{Y} 2$ and $\mathrm{Y} 10$. The adjusted IRRs (95\%Cl) of developing dementia were $1.06(0.72,1.60), 1.28(0.82,2.00)$ and $0.89(0.68,1.17)$ for benzodiazepines, ACB3 and ACB12 ever-users compared with non-users. For recurrent users the respective IRRs were $1.30(0.79,2.14), 1.68(1.00,2.82)$ and $0.95(0.71$, 1.28). ACB3 ever-use was associated with dementia among those with $Y 2$ MMSE $>25$ (IRR $=2.28$ [1.32-3.92]), but not if Y2 MMSE $\leq 25(\mathrm{IRR}=0.94[0.51-1.73])$.

Conclusions: Neither benzodiazepines nor ACB12 medications were associated with dementia. Recurrent use of ACB3 anticholinergics was associated with dementia, particularly in those with good baseline cognitive function. The longterm prescribing of anticholinergics should be avoided in older people.
\end{abstract}

Keywords: Alzheimer disease, Cognition, Dementia, Cohort study, Benzodiazepines, Cholinergic antagonists

\section{Background}

Dementia prevention is a public health priority. No disease modifying treatment for dementia exists, but dementia risk and progression can be modified by changing exposure to risk factors affecting any aspect of long-term brain health [1]. Identifying such risk factors is important for dementia prevention and cognitive health.

\footnotetext{
* Correspondence: Kathryn.richardson@uea.ac.uk

'School of Health Sciences, University of East Anglia, Norwich NR4 7TJ, UK Full list of author information is available at the end of the article
}

Long-term use of several classes of medications have been suggested to increase future dementia risk. Medications with anticholinergic activity (henceforth anticholinergics), benzodiazepines and related non-benzodiazepine derivatives have come under particular scrutiny owing to their well-known short-term cognitive effects [2] and the high prevalence of their long-term use among middle aged and older people [3, 4].

Anticholinergics are successfully used in the treatment of many conditions such as urinary incontinence, Parkinson's disease, depression, and epilepsy. Anticholinergics can adversely affect cognition [2]; guidelines suggest they

(c) The Author(s). 2019 Open Access This article is distributed under the terms of the Creative Commons Attribution 4.0 International License (http://creativecommons.org/licenses/by/4.0/), which permits unrestricted use, distribution, and reproduction in any medium, provided you give appropriate credit to the original author(s) and the source, provide a link to the Creative Commons license, and indicate if changes were made. The Creative Commons Public Domain Dedication waiver (http://creativecommons.org/publicdomain/zero/1.0/) applies to the data made available in this article, unless otherwise stated. 
are to be avoided among frail older people [5] or those with dementia [6]. Over the past decade, prolonged exposure to anticholinergics has been linked to long term cognitive decline or dementia [7-12]. Many medicines beyond those typically regarded as anticholinergics may have mild anticholinergic effects and it has been suggested that the cumulative long term use of many such medications may increase dementia risk [11]. Depending on their definition, anticholinergic medications are used by $10-50 \%$ of the middle aged and older population at any time [13, 14].

Benzodiazepines and non-benzodiazepine derivatives are primarily used to treat anxiety or insomnia. Short term cognitive effects due to their sedating action are well recognised. Although long-term use is not recommended many people use regularly benzodiazepines and related medicines for years or decades [3]. Estimates of the effect of benzodiazepine use on long term cognitive decline and dementia have been mixed [15-22].

For both benzodiazepines and anticholinergics, several methodological biases exist in the published studies including first the lack of longitudinal observational window with a clear baseline measurement of cognitive and functional status of the population at risk; second the absence of gold standard measurement of the dementia incidence; third, no precise baseline measurement of the exposure variable (benzodiazepine and anticholinergic use); and finally the limitation of observational studies in resolving protopathic bias, whereby medication use might be prescribed for symptoms at the very early stages of dementia, inducing an association between medication use and later dementia diagnosis.

We have previously demonstrated association between cognitive decline and anticholinergic use between baseline and 2-year follow-up assessments of the Medical Research Council Cognitive Function and Ageing Study (MRC CFAS) [9]. Here we extend this analysis to examine dementia incidence at 10 year follow-up, with respect to patterns of anticholinergic and benzodiazepine use at the baseline and 2-year follow-up assessments.

\section{Methods}

\section{Setting}

The MRC CFAS is a population based, prospective, multicentre cohort study in England and Wales specifically designed to estimate the prevalence, risk factors and course of dementia. The study design has been described elsewhere [23]; (see also www.cfas.ac.uk for full details).

In brief, 13,004 participants, age 65 and older, from Cambridgeshire, Gwynedd, Newcastle, Nottingham and Oxford, were recruited with baseline interviews (Y0) conducted between 1991 and 1993. All individuals still alive and traceable were invited to be re-interviewed at two years (Y2) and 10 years (Y10) after baseline. At each wave, participants were questioned about sociodemographic factors, lifestyle, physical and mental health (including self-reported insomnia, measures of anxiety and depression) and completed a cognitive battery and in-home medication inventory. For the present analysis, we included all those who participated at Y2 with no study diagnosis of dementia at $\mathrm{Y} 0$ or at $\mathrm{Y} 2$, and measured incident dementia as an outcome at Y10.

\section{Outcome assessment}

At $\mathrm{YO}$ and $\mathrm{Y} 2$ the study diagnosis of dementia was made using a two-phase process (Fig. 1). An initial screening interview was administered to all participants. A stratified subsample of $20 \%$, including all of those with cognitive impairment, but also including healthy participants then underwent a thorough assessment using the Automated Geriatric Examination for Computer Assisted Taxonomy (AGECAT) algorithm to make a study diagnosis of dementia [23-26]. AGECAT produces a score of between 0 and 5 . Dementia was defined as AGECAT scores $\geq 3$ which is equivalent to dementia as diagnosed by DSM-III-R [24]. All surviving participants underwent the full assessment at Y10.

For those who underwent a screen interview but were not selected to undergo the assessment we imputed the Y2 dementia status based on cognitive screen scores (using a multiple imputation). This procedure identified that there were possibly a small number of cases of dementia among the screen-only sample, but these were only very rarely seen among those surviving Y10 sample. Hence our primary analysis assumed no prevalent dementia cases among the Y2 screen-only participants; participants who were imputed to have dementia at baseline were excluded in a sensitivity analysis.

\section{Medication exposures}

During each interview participants were asked to provide details of all medication currently being used, either prescribed or bought over-the-counter. These were recorded using UK National Health Service Read codes. Packaging was checked and proxy respondents supplied medication information if participants were unable to do so. Previous studies in older population have demonstrated self-reported medication data gathered in this way to be mostly in moderate-good agreement with prescription data records [27].

All medications were coded according to the Anticholinergic Cognitive Burden (ACB) scale [28]. In summary, medications with serum anticholinergic activity or in vitro affinity to muscarinic receptors but with no known clinically relevant negative cognitive effects are scored 1 on the scale, while drugs with established and clinically relevant anticholinergic effects are scored 2 based on blood-brain penetration and 3 if also have reported associations with delirium. All other drugs are 


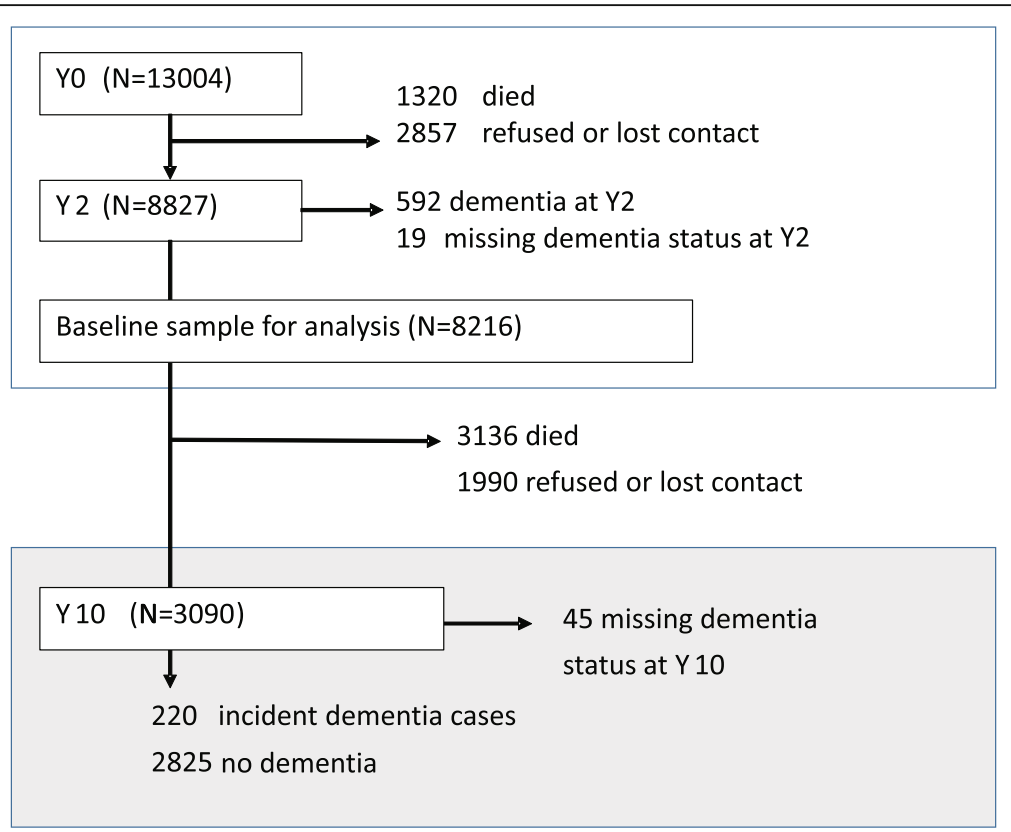

Fig. 1 Flow of participants included in the current analysis through the MRC Cognitive Function and Ageing Study. See www.cfas.ac.uk for the full design of the Cognitive Function and Ageing Studies

scored 0 . Very few medications were classed as having an ACB score of 2, so we created binary exposure variables for ACB12 (use of any medications scoring 1 or 2) and ACB3 (use of any medications scoring 3). A total $\mathrm{ACB}$ sum score, and a variable corresponding to the sum of ACB12 drugs only was also created. Each of these exposures was determined independently at Y0 and $\mathrm{Y} 2$.

Similarly for benzodiazepines, a binary variable (BZD) corresponding to taking any benzodiazepine or nonbenzodiazepine derivative (hypnotics such as zopiclone also known as Z-drugs) was created at both Y0 and Y2.

For each group (BZD, ACB12 and ACB3) participants were then classified as being an 'ever-user' (if there was any use at $\mathrm{Y0}$ or $\mathrm{Y} 2$ ), and then sub-classified as a 'recurrent user' (use at $\mathrm{YO}$ and Y2), new user (only at Y2), or as a discontinuing user (only at $\mathrm{Y0}$ ).

\section{Covariates}

We selected covariates that might have a confounding effect between the use of benzodiazepines or anticholinergics and incident dementia. We included demographic variables of sex, age, education ( $\leq 9$ years, $\geq 10$ years), social class (measured by prior occupation as manual vs non manual), centre of recruitment, and study arm (screen or assessment), variables that are indicators for ACB3 or BZD use, early symptoms of dementia or known to be associated with dementia (reporting having suffered stroke, Parkinson disease, epilepsy, sleep problems, anxiety, depression or being diagnosed depression at either Y0 or Y2, as binary variables), self- reported health (excellent/good; fair/poor) at Y2 and cognition related variables.

Pre-existing cognitive impairment and ongoing cognitive decline are the most important potentially confounding factors, these were measured by the Mini-Mental State Examination (MMSE) at Y2 $(\leq 25,>25)$, the decrease in MMSE scores between $\mathrm{Y} 0$ and $\mathrm{Y} 2(<1,1,2, \geq 3$ points), the MMSE orientation sub-score at $\mathrm{Y} 2(<9,9 / 10)$ and self-perceived change in memory function between recruitment and 2 years (No change or better vs worse). Disability at $\mathrm{Y} 2$ was classified using the Townsend disability scale as either no impairment, any impairment in instrumental activities of daily living or any impairment in basic activities of daily living [29].

\section{Statistical analyses}

Separate univariable Poisson regression models with Huber-White robust standard errors were used to estimate incidence rate ratios (IRR) for the association between each potential predictor variable and incident dementia at Y10 [30]. 95\% confidence intervals are reported for all estimates.

In multivariable analysis we additionally included each of the three ever-use variables (where they were not the exposure of interest) and the demographic, health and cognition related variables mentioned above.

We carried out pre-planned stratified analyses of the main 'ever-use' models by year of birth ( $\leq 1919$ vs 1920 onwards), sex and MMSE score at Y2 $(>25, \leq 25)$. The 
threshold for cognitive function and age were chosen as they reflect the stratification of the original CFAS study sampling.

As expected in this population there was substantial loss to follow-up between Y2 and Y10 caused by drop out and death. Inverse probability weights were used to adjust for non-response at Y10 and loss of contact between Y2 and Y10 or refusal to participate at Y10, conditional on having survived. These weights were calculated using a logistic regression model for being successfully re-assessed at Y10 (conditional on surviving to Y10) including the main effects of all exposures (BZD, $\mathrm{ACB} 12$ and $\mathrm{ACB} 3)$, covariates and the interactions between exposures and sex and MMSE at Y2.

STATA 14.1 was used for all analysis.

\section{Sensitivity analyses}

We carried out three sensitivity analyses to test the impact of modelling assumptions or analytical choices on our results. First, we excluded potentially mediating or colliding variables: MMSE at Y2, change in MMSE (Y0 to Y2), MMSE orientation sub-score at Y2, disability, and arm of the study. Second, we used multiple imputation to identify screen-only participants with dementia at baseline based on their demographic information and cognitive scores as described above, and excluded them from each imputed analysis. Finally, we took into account the possibility that higher mortality rates among older people taking anticholinergics or benzodiazepines and related medications might suppress our estimates of dementia incidence in this group via inverse probability weights calculated using on the probability of death or drop-out (rather than drop-out alone) between Y2 and Y10 based on baseline factors.

\section{Results}

See Fig. 1 for participant flow through the study. From the 13,004 participants recruited to MRC CFAS at Y0, 8216 were interviewed at $\mathrm{Y} 2$, did not have dementia or unknown dementia status at Y2 and so form the baseline sample for our incidence analysis. Of these, 3136 died and 1990 were lost to follow up before Y10. At Y10, we excluded a further 5 participants classified as having dementia at Y0 but not Y10 and 45 with unknown dementia status at Y10, leaving 220 people with incident dementia and 2825 people without incident dementia included in the study.

Table 1 shows participant characteristics stratified by follow-up status. Those who developed dementia by Y10 were older, had lower cognitive function at Y2 (mean MMSE 24 vs 27), more disability (ADL-IADL $22 \%$ vs $7 \%$ ), fewer years of education ( $\geq 10$ years $29 \%$ vs $44 \%$ ) and were substantially more likely to report worsening memory from recruitment to 2 -year follow up (49\% vs $27 \%$ ) and poorer health (32\% vs $21 \%)$.

\section{Medication use}

A breakdown of baseline exposures by 10-year follow-up status is shown in Table 1. Full details of drug use are in Additional file 1 . Among those surviving to 10 years, $7.5 \%$ reported ever use of a BZD (short-acting $4.2 \%$, long-acting 3.7\%). Hypnotic BZD were used by $5.9 \%$ with $1.9 \%$ using anxiolytics. The most commonly reported BZDs were Temazepam (47\% of BZDs reported), Nitrazepam (30\%) and Diazepam (15\%). Nonbenzodiazepine Z-drug use was rare in this cohort (prevalence of $0.4 \%$ ).

Use of ACB3 at baseline or 2-year follow-up was reported by $5.6 \%$ of the surviving sample; $2.3 \%$ were recurrent users. The majority of ACB3 drugs were antidepressants (3.8\% of the surviving sample; corresponding to $69 \%$ of ACB3 medications), urologicals (0.7\% reported ever use among the sample), gastrointestinal $(0.6 \%)$, antipsychotics $(0.5 \%)$, antihistamines $(0.3 \%)$ and Parkinsonian drugs (0.1\%). The most common ever-use ACB3 medications were the antidepressants: amitriptyline $(22 \%$ of ACB3) and dosulepin (22\% and of ACB3).

In total, $53 \%$ of the surviving sample reported ACB1 or ACB2 at baseline or 2-year follow-up, with $34 \%$ reporting ACB1 or ACB2 use at both waves.

Although Y10 medication is not considered an exposure in our study, we compared Y10 to Y0 and Y2 medication to understand to what extent medication use was likely to have continued in the overall study sample. Medication use at Y10 was highly correlated with use at Y0 and Y2 (see Additional file 2) with around 60\% of 'recurrent' users at $\mathrm{Y} 0$ and $\mathrm{Y} 2$ reported use of each class at Y10. This suggests that in many cases use at Y0 and Y2 is likely to reflect repeated use during the follow-up period as opposed to being one-off exposures.

\section{Dementia incidence}

Table 2 describes incident dementia in our sample as well as the unadjusted and adjusted incidence rate ratios (aIRR). After weighting, 9.5\% $(N=220)$ of participants had a study diagnosis of dementia at Y10; 14.5, 15.4 and 10.5\% for BZD, ACB3, ACB12 ever-users and 16.0, 18.6 and $10.7 \%$ for recurrent users, respectively.

Adjusted IRRs for dementia at Y10 were $1.06(95 \% \mathrm{CI}$ $0.72,1.60)$ for any BZD use, 1.28 (95\% CI $0.82,2.00)$ for any $\mathrm{ACB} 3$ and 0.89 (95\%CI 0.68 1.17) for any ACB12 use. Recurrent use was associated with IRRs of 1.30 $(95 \%$ CI $0.79,2.14)$ for BZD, 1.68 (95\%CI 1.00, 2.82) for ACB3 and 0.95 (95\%CI 0.71, 1.28) for ACB12.

There was no evidence for an increase in dementia risk with increasing total $\mathrm{ACB}$ score at each wave, or with the number of ACB1 or ACB2 medications used. 
Table 1 Participant characteristics stratified by $Y 10$ follow-up status and dementia outcome

\begin{tabular}{|c|c|c|c|c|}
\hline Characteristic & $\begin{array}{l}\text { Dementia at } \mathrm{Y} 10 \\
(\mathrm{n}=220)\end{array}$ & $\begin{array}{l}\text { No dementia at } Y 10 \\
(n=2825)\end{array}$ & $\begin{array}{l}\text { Lost to follow up between Y2 and } \\
\text { Y10 }(n=1990)\end{array}$ & $\begin{array}{l}\text { Died between } Y 2 \text { and } Y 10 \\
(n=3136)\end{array}$ \\
\hline Female & $163(77.3)$ & $1675(61.2)$ & $1315(66.1)$ & $1630(52.0)$ \\
\hline Mean age (SD) & $77.1(7.0)$ & $72.0(10.0)$ & $73.8(6.1)$ & $76.9(6.7)$ \\
\hline Educated for $\geq 10$ years & $68(29.3)$ & $1311(43.9)$ & $754(37.9)$ & $1127(35.9)$ \\
\hline Manual occupation & $132(61.7)$ & $1359(50.1)$ & $1088(54.7)$ & $1751(55.8)$ \\
\hline CFAS assessment arm & $84(51.0)$ & $594(27.4)$ & $814(40.9)$ & $999(31.9)$ \\
\hline \multicolumn{5}{|l|}{ Y2 MMSE } \\
\hline$\leq 21$ & $30(22.6)$ & $57(3.5)$ & $190(9.6)$ & $381(12.2)$ \\
\hline $22-25$ & $76(38.4)$ & $432(20.0)$ & $552(27.7)$ & $845(27)$ \\
\hline $26-30$ & $114(39.0)$ & $2336(76.5)$ & $1196(60.1)$ & $1847(58.9)$ \\
\hline \multicolumn{5}{|l|}{ Decline in MMSE between $Y 0$ and $Y 2$} \\
\hline No decline / improvement & $100(41.1)$ & $1592(55.0)$ & $988(49.6)$ & $1444(46.0)$ \\
\hline 1 point & $34(13.1)$ & $529(17.6)$ & $302(15.2)$ & $487(15.5)$ \\
\hline 2 points & $34(13.6)$ & $350(11.8)$ & $220(11.1)$ & $364(11.6)$ \\
\hline$\geq 3$ points & $50(29.0)$ & $326(14.2)$ & $402(20.2)$ & $729(23.2)$ \\
\hline \multicolumn{5}{|l|}{ Disability } \\
\hline None & $121(47.1)$ & $2336(80.0)$ & $1386(69.6)$ & $1562(49.8)$ \\
\hline IADL impairment & $65(30.2)$ & $350(13.1)$ & $331(16.6)$ & $672(21.4)$ \\
\hline ADL impairment / unclassified & $34(22.7)$ & $139(7.0)$ & $273(13.7)$ & $902(28.8)$ \\
\hline $\begin{array}{l}\text { Self-reported memory decline } \\
\text { (Y0 to Y2) }\end{array}$ & $110(48.7)$ & $774(27.4)$ & $592(29.7)$ & $1152(36.7)$ \\
\hline Fair/poor self-reported health & $66(31.7)$ & $529(21.2)$ & $523(26.3)$ & $1174(37.4)$ \\
\hline \multicolumn{5}{|l|}{ Comorbidity $^{a}$} \\
\hline Sleep disturbance & $56(26.5)$ & $606(22.6)$ & $507(25.5)$ & $902(28.8)$ \\
\hline Diagnosed depression & $22(11.0)$ & $309(11.3)$ & $216(10.9)$ & $290(9.2)$ \\
\hline Consulted GP for depression & $31(15.8)$ & $388(14.4)$ & $282(14.2)$ & $387(12.3)$ \\
\hline Consulted GP for anxiety & $28(11.8)$ & $242(8.5)$ & $186(9.3)$ & $228(7.3)$ \\
\hline \multicolumn{5}{|l|}{ BZD use } \\
\hline None & $195(86.6)$ & $2623(91.7)$ & $1763(88.6)$ & $2726(86.9)$ \\
\hline Any ${ }^{e}$ & $25(13.5)$ & $202(8.3)$ & $222(11.2)$ & $391(12.5)$ \\
\hline $\mathrm{New}^{f}$ & $5(2.2)$ & $43(1.9)$ & $49(2.5)$ & $92(2.9)$ \\
\hline Discontinuing ${ }^{g}$ & $6(3.2)$ & $51(2.0)$ & $57(2.9)$ & $95(3.0)$ \\
\hline Recurrent $^{\mathrm{h}}$ & $14(8.1)$ & $108(4.4)$ & $116(5.8)$ & $204(6.5)$ \\
\hline \multicolumn{5}{|l|}{ ACB3 use ${ }^{c}$} \\
\hline None & $198(89.8)$ & 2677 (94.1) & 1842 (92.6) & $2831(90.3)$ \\
\hline Any $^{e}$ & $22(10.2)$ & $148(5.9)$ & $143(7.2)$ & $286(9.1)$ \\
\hline $\mathrm{New}^{f}$ & $5(1.8)$ & $55(2.2)$ & $58(2.9)$ & $112(3.6)$ \\
\hline Discontinuing ${ }^{9}$ & $5(3.6)$ & $35(1.5)$ & $43(2.2)$ & $78(2.5)$ \\
\hline Recurrent $^{\mathrm{h}}$ & $12(4.9)$ & $58(2.2)$ & $42(2.1)$ & $96(3.1)$ \\
\hline \multicolumn{5}{|l|}{ ACB12 use $e^{d}$} \\
\hline None & $85(41.0)$ & $1353(47.3)$ & 908 (45.6) & $972(31)$ \\
\hline Any ${ }^{e}$ & $135(59.0)$ & $1472(52.7)$ & $1077(54.1)$ & $2145(68.4)$ \\
\hline $\operatorname{New}^{f}$ & $34(16.0)$ & $321(11.4)$ & $210(10.6)$ & 419 (13.4) \\
\hline Discontinuing ${ }^{9}$ & $11(4.5)$ & $209(7.9)$ & $175(8.8)$ & $327(10.4)$ \\
\hline Recurrent $^{\mathrm{h}}$ & $90(38.5)$ & $942(33.5)$ & $692(34.8)$ & 1399 (44.6) \\
\hline
\end{tabular}


Number of participants (percentages) given unless specified otherwise. Percentages of participants at $Y 10$ are weighted for attrition due to non-response at $Y 10$ and loss of contact between $\mathrm{Y} 2$ and $\mathrm{Y} 10$

Abbreviations: CFAS Cognitive Function and Ageing Study, SD standard deviation, MMSE Mini-Mental State Examination, IADL Instrumental Activities of Daily Living, ADL Activities of Daily Living, GP General Practitioner

${ }^{a}$ Any record of specific comorbidity at the $\mathrm{Y} 0$ or $\mathrm{Y} 2$ assessment

bUse of benzodiazepines or Z-drugs

'Use of drugs scoring 3 on the Anticholinergic Cognitive Burden scale

dUse of drugs scoring 1 or 2 on the Anticholinergic Cognitive Burden scale

e-h Drug use categories are

None: no use at $\mathrm{YO}$ or $\mathrm{Y} 2$;

Any: Use at $\mathrm{YO}$ or $\mathrm{Y} 2$;

New: Use at $Y 2$ but not $Y 0$

Discontinuing: Use at $\mathrm{Y} 0$ but not $\mathrm{Y} 2$

Table 2 Attrition-weighted unadjusted and multivariable adjusted incidence rate ratios for the association between benzodiazepine and anticholinergic medication use and incident dementia

\begin{tabular}{|c|c|c|c|c|c|}
\hline \multirow{2}{*}{$\begin{array}{l}\text { Exposure and pattern } \\
\text { of use }\end{array}$} & \multicolumn{3}{|c|}{ Dementia incidence } & \multirow{2}{*}{$\begin{array}{l}\text { Unadjusted } \\
\text { IRR }(95 \% \mathrm{Cl})\end{array}$} & \multirow{2}{*}{$\begin{array}{l}\text { Adjusted }^{\mathrm{b}} \text { IRR } \\
(95 \% \mathrm{Cl})\end{array}$} \\
\hline & Cases & Total & $\%^{a}$ & & \\
\hline \multicolumn{6}{|l|}{ BZD use } \\
\hline None & 195 & 2819 & 9.0 & 1 (Ref.) & 1 (Ref.) \\
\hline Any & 25 & 227 & 14.5 & $1.61(1.06,2.46)$ & $1.06(0.72,1.60)$ \\
\hline New & 5 & 48 & 11.1 & $1.23(0.51,2.96)$ & $0.65(0.27,1.60)$ \\
\hline Discontinuing & 6 & 57 & 14.1 & $1.57(0.74,3.35)$ & $1.06(0.53,2.14)$ \\
\hline Recurrent & 14 & 122 & 16.0 & $1.78(1.02,3.12)$ & $1.30(0.79,2.14)$ \\
\hline \multicolumn{6}{|l|}{ ACB3 use } \\
\hline None & 198 & 2876 & 9.1 & 1 (Ref.) & 1 (Ref.) \\
\hline Any & 22 & 170 & 15.4 & $1.70(1.09,2.65)$ & $1.28(0.82,2.00)$ \\
\hline New & 5 & 60 & 7.9 & $0.88(0.37,2.09)$ & $0.87(0.34,2.22)$ \\
\hline Discontinuing & 5 & 40 & 20.1 & $2.22(0.96,5.14)$ & $1.19(0.53,2.68)$ \\
\hline Recurrent & 12 & 70 & 18.6 & $2.05(1.18,3.56)$ & $1.68(1.00,2.82)$ \\
\hline \multicolumn{6}{|l|}{ ACB3 subclass } \\
\hline Not antidepressants & 6 & 53 & 13.6 & $1.50(0.68,3.32)$ & $1.74(0.84,3.62)$ \\
\hline Antidepressants & 16 & 117 & 16.1 & $1.78(1.06,2.98)$ & $1.16(0.69,1.94)$ \\
\hline \multicolumn{6}{|l|}{$A C B 1$ or $A C B 2$ use } \\
\hline None & 85 & 1438 & 8.3 & 1 (Ref.) & 1 (Ref.) \\
\hline Any & 135 & 1609 & 10.5 & $1.26(0.95,1.67)$ & $0.89(0.68,1.17)$ \\
\hline New & 34 & 355 & 12.8 & $1.54(1.02,2.33)$ & $1.14(0.79,1.63)$ \\
\hline Discontinuing & 11 & 220 & 5.6 & $0.68(0.36,1.27)$ & $0.36(0.19,0.69)$ \\
\hline Recurrent & 90 & 1033 & 10.7 & $1.29(0.95,1.75)$ & $0.95(0.71,1.28)$ \\
\hline \multicolumn{6}{|l|}{ ACB sum score } \\
\hline Total ACB score (per point) & & & & $1.07(1.03,1.13)$ & $1.00(0.94,1.06)$ \\
\hline ACB12 score (per point) & & & & $1.06(1.00,1.13)$ & $0.97(0.90,1.04)$ \\
\hline ACB3 score (per point) & & & & $1.10(1.02,1.19)$ & $1.06(0.98,1.15)$ \\
\hline
\end{tabular}

Abbreviations: IRR Attrition-weighted unadjusted incidence rate ratio, alRR Attrition-weighted adjusted incidence rate ratio, $C l$ confidence-interval, $A C B$ Anticholinergic Cognitive Burden, ACB1 use of a medicine with an ACB score of 1 . Scores correspond to possibly anticholinergic (score 1) probably anticholinergic (score 2) definitely anticholinergic (score 3 )

a represents weighted incidence

${ }^{b}$ Adjusted for sex, age, education ( $\leq 9$ years, $\geq 10$ years), social class (manual vs non manual), residential accommodation, centre of recruitment, study arm (screen or assessment), health conditions at Y0 or Y2 (stroke, Parkinson disease, epilepsy, sleep problems, anxiety, depression), self- reported health (excellent/good; fair/ poor) at $Y 2$, Disability at $Y 2$ (no impairment, impairment in instrumental activities of daily living, or impairment in basic activities of daily living), Mini-Mental State Examination (MMSE) at Y2 $(\leq 25,>25)$, MMSE orientation sub-score at $Y 2(<9,9 / 10)$, decrease in MMSE score between $Y 0$ and $Y 2(<1,1,2, \geq 3$ points), and selfperceived change in memory function between $\mathrm{Y} 0$ and $\mathrm{Y} 2$ (No change or better vs worse) 
No significant association was found between dementia and ever-use of short or medium-acting, long-acting, hypnotic or anxiolytic BZDs, or for anti-depressant or 'other' anticholinergics although numbers in these subgroups were small (results not shown).

\section{Stratified analysis}

Stratified analyses are shown in Table 3. The effect of ACB3 was restricted to those with good baseline cognitive function (ever-users aIRR: 2.28, 95\%CI 1.32, 3.92), whereas no such association was seen among the group with impaired cognition (ever-users aIRR: 0.94, 95\% CI: 0.51-1.73). Those with poor cognitive function (MMSE $\leq 25$ at Y2) had a dementia incidence rate of around $21 \%$ irrespective of anticholinergic use (21.3\%; 97 of 500 among never-users vs 21.8\%; 9 of 46 for ever-users), while for those with good cognitive function (MMSE $>25$ at Y2) the Y10 dementia incidence rate was $11.1 \%$ (13 of 124) for ACB3 ever-users and 4.7\% (101 of 2326) for never-users (Additional file 3). This is supported by a statistically significant interaction effect $(p=0.02)$. No other significant subgroup differences were found.

\section{Sensitivity analyses}

Results from the sensitivity analyses are shown in Additional file 4. No changes were seen after removing imputed possible dementia cases at baseline or 2-year follow-up. However, after excluding baseline disability and cognition related variables from multivariable regression there was an increase in the effects of any ACB3 use and recurrent use with aIRRs 1.55 (95\%CI $1.04,2.32)$ and $2.02(95 \% \mathrm{CI} 1.21,3.39)$, respectively. No main changes were observed when using weights to adjust for mortality or after carrying out a competing risk analysis (results not shown). In analysis stratified by cognitive score, there is no change to main findings in sensitivity analysis; for example when using inverse probability weights to adjust for attrition by death or other loss to follow up the association between baseline
ACB3 use and incident dementia among those with MMSE $>25$ at $\mathrm{Y} 2$ is aIRR $=2.24$ (95\% CI: 1.24-4.06) compared to IRR $=1.01(0.55-1.87)$ among those with Y2 MMSE< 25.

\section{Discussion}

In a cohort study with 10-year follow-up we did not find any evidence of an increase in risk of dementia associated with the use benzodiazepines or anticholinergics scoring ACB1 or ACB2. We did find a statistically significant increase in dementia risk among recurrent users of ACB3 anticholinergics and also an association between ACB3 anticholinergics use and dementia risk among the subgroup with good baseline cognitive function, suggesting that effects might more apparent in different subgroups of the older population.

\section{Benzodiazepines}

Previous studies on the effect of benzodiazepines have been inconsistent, with some large and apparently high quality studies showing a clear effect of benzodiazepine use on dementia incidence [16-18, 20, 21], but others finding no effect $[15,19,22]$. There is no readily apparent difference between these studies in design that explains this inconsistency, although possible explanations include selection biases into electronic health record databases, differing methods of ascertaining benzodiazepine use, such as duration, dose and chronicity and the measurement of dementia outcome [15], or the differing profile of benzodiazepine use [31], population characteristics across studies or the manner in which each study was able to control for covariates. There was insufficient use of Z-drugs among our cohort to draw any conclusions regarding their effects on dementia incidence.

\section{Strong anticholinergics}

Our estimate of the effect of ACB3 anticholinergics on dementia incidence was not statistically significant, but is consistent with recent effect estimates from analyses

Table 3 Attrition-weighted adjusted incidence rate ratios for benzodiazepine and anticholinergic medication use and incident dementia, stratified by cognition, sex and age

\begin{tabular}{llll}
\hline \multirow{2}{*}{ Subgroup } & \multicolumn{4}{l}{ Incidence rate ratio (95\% confidence interval) by exposure } & Any ACB12 \\
\cline { 2 - 4 } & Any Benzodiazepines & Any ACB3 & $0.99(0.68,1.43)$ \\
\hline MMSE at Y2 $>25$ & $0.72(0.35,1.50)$ & $2.28(1.32,3.92)^{*}$ & $0.78(0.54,1.12)$ \\
MMSE at Y2 $\leq 25$ & $1.23(0.74,2.06)$ & $0.94(0.51,1.73)$ & $1.11(0.66,1.89)$ \\
Male & $0.29(0.06,1.31)$ & $2.06(0.78,5.46)$ & $0.85(0.63,1.16)$ \\
Female & $1.17(0.77,1.78)$ & $1.24(0.77,2.01)$ & $1.57(0.82,3.00)$ \\
Younger (born 1920-1929) & $1.31(0.52,3.27)$ & $1.16(0.45,3.01)$ & $0.77(0.58,1.03)$ \\
Older (born before 1920) & $1.06(0.69,1.61)$ & $1.27(0.78,2.09)$ & \\
\hline
\end{tabular}

${ }^{*} p<0.01$

Abbreviations: MMSE Mini-Mental State Examination, ACB Anticholinergic Cognitive Burden, ACB12 use of a medicine with an ACB score of 1 or 2. Scores correspond to possibly anticholinergic (score 1), probably anticholinergic (score 2) and definitely anticholinergic (score 3 ). Number of observations and adjusted percentage in each group are reported in Additional file 3 
of electronic medical records [7, 32]. However, in planned subgroup analyses we observed a borderline significant increased dementia risk in recurrent users of ACB3 anticholinergics, defined as those participants who reported anticholinergic use at both baseline and twoyear follow-up, more likely to reflect a longer term or continuous anticholinergic load. This is consistent with the hypothesis that long-term as opposed to one-off use is needed to increase dementia risk.

Consistent with our work, previous studies have consistently reported associations between anticholinergic use and dementia incidence, with a greater effect seen among prevalent (as opposed to new users) or long-term recurrent users, with some studies reporting a dose effect with increasing risk at higher doses [7, 32]. New use or short term use has consistently not been associated with risk of developing dementia [8]. Similar results have been observed for studies focussing on cognitive change instead of dementia or MCI outcomes and in neuropathology studies [33, 34].

We stratified our analysis by baseline cognitive function to test the hypothesis that the effect is only seen among people with an existing cognitive impairment, reflecting possible protopathic bias. In fact the reverse was observed, the effect was restricted to those with good baseline cognitive function. It is possible that this reflects increased attrition among the more cognitively frail using anticholinergics, however this finding is not affected by using a weight that corrects for attrition due to death, and in any case this results demonstrates that the increase in dementia incidence associated with anticholinergics is not restricted to those with existing cognitive impairment or those with incipent dementia.

Anticholinergics represent a broad class of medications that act on different systems, and it is possible that different anticholinergics have different long term effects on brain health [12]. Disaggregation of anticholinergic classes may also help to identify possible confounding by indication or protopathic bias. Our study suggests that anticholinergics other than antidepressants have a stronger link with incident dementia than do anticholinergic antidepressants after adjustment for confounding factors, but owing to small numbers estimates of the effects of subclasses are very imprecise $[7,12]$.

\section{Anticholinergics with score of 1 or 2}

While ACB3 anticholinergics are used by only $3-5 \%$ of the older population at any time, up to $50 \%$ are using one or more of the much wider group that are considered 'possibly' anticholinergic (score of 1), and any effect of these medications on dementia incidence would have a great public health significance [9]. Our finding that the number of ACB12 anticholinergics used is not associated with future incident dementia agrees with our previous analysis of cognitive change between baseline and 2 years [9] and previous studies that have considered these groups separately $[12,35,36]$. The number of medications classified as ACB2 is very small and this effect estimate is largely dominated by the effect of medications classified ACB1. Findings from the Baltimore Longitudinal Study of Ageing suggest an increase in the risk of 'Alzheimer's disease or MCI' with increasing use of 'possible' anticholinergics, with an associated increase in cortical atrophy, although there was no effect of definite anticholinergic (score of 3) use suggesting that anticholinergic properties of these drugs may not underlie the effect [37].

\section{Strengths and limitations}

Our study has several important strengths and limitations. By using the first two waves of MRC CFAS (years 0 and 2) as the baseline and dementia at 10-year followup as the outcome we could identify the long-term effect of different patterns of uses of medications in a population-representative cohort. We did not measure medication use or dementia diagnoses occurring between assessments, or the diagnoses for those who dropped out before Y10. Although the high concordance between medications used at Y0, Y2 and Y10 suggests that use may have been continual during the follow up period in many cases, we have no direct evidence for this. Medication use was based on self-report and adherence was not formally assessed; although there is no gold standard method for measuring adherence to medication [38]. Dementia was measured using a validated algorithm, and thus any bias due to outcome ascertainment is reduced compared to studies relying on a recorded diagnosis dementia which will significantly under represent true dementia incidence [39].

Despite the large sample size of MRC CFAS $(n=13,004)$, the numbers using benzodiazepines or anticholinergics with score ACB3 during the first two waves and developing incident dementia by Y10 are relatively small. Estimating effects for subgroups is difficult. Attrition over 8 years was typical of that seen in comparable studies of ageing, and we applied inverse probability weighting based on exposures and baseline cognitive scores to adjust for differential drop-out. Use of inverse probability weights assumes that loss to follow-up or death was not differential with respect to unmeasured confounders or to the outcome. Our findings might be biased if the interaction between medication use and dementia has a specific association with drop-out that could not be attributed to either factor alone or the interaction between exposure and pre-existing cognitive impairment.

We controlled for many relevant potential confounders, in particular for many of the indications for anticholinergics and benzodiazepines. We could not 
control for urinary incontinence or obesity as this was not routinely recorded, however the anticholinergic urologicals were rarely used among this cohort. Mental health disorders apart from depression and anxiety were also not routinely recorded. Adjusting for recent cognitive decline and observing the effect among those with good cognitive function at $\mathrm{Y} 2$ helps to exclude the possibility of protopathic bias due to reverse causation.

\section{Conclusions}

We found no evidence that benzodiazepines are associated with dementia incidence but we cannot rule out an effect as the number of benzodiazepine users in our study was relatively small. Consistent with previous studies we found an increase in dementia incidence associated with the recurrent use of anticholinergics with an ACB score of 3, particularly among those with good baseline cognitive function. This should be treated with caution owing to small sample size but when considered alongside the growing body of evidence from cohort studies and administrative data sources suggests that at least some anticholinergic medications could increase the risk of future dementia. The prevalence of anticholinergic medication use remains high among middle aged and older people, making this a potentially important modifiable risk factor for dementia. Future research should focus on more carefully establishing the mechanism by which this occurs, whether the effect is reversed by medication cessation and whether specific anticholinergic medication or classes of medication confer the greatest risk and among which subgroups of the population.

\section{Supplementary information}

Supplementary information accompanies this paper at https://doi.org/10. 1186/s12877-019-1280-2.

Additional file 1. Baseline use and 2-year patterns of use of benzodiazepines and anticholinergics of participants with 10-year followup (unweighted percentage).

Additional file 2: Table S1. a Use of benzodiazepines/Z-drugs at 10year follow-up stratified by pattern of benzodiazepine/Z-drug use from Y0 to Y2 (percentages are unweighted). b Use of anticholinergics with score of 3 at $Y 10$ stratified by pattern of anticholinergic use from $Y 0$ to Y2 (percentages are unweighted).

Additional file 3. Incidence risk ratios for benzodiazepine and anticholinergic medication use and dementia, weighted for attrition and stratified by MMSE, sex and age.

Additional file 4. Adjusted incidence rate ratios ( $95 \% \mathrm{Cl}$ ) for dementia from the sensitivity analyses of the main findings using fewer covariates, different dementia exclusion criteria, or different attrition weights.

\section{Abbreviations}

ACB: Anticholinergic cognitive burden; ACB12: Anticholinergics with score 1 or 2; ACB3: Anticholinergics with score 3; AGECAT: Automated geriatric examination for computer assisted taxonomy; alRR: Adjusted incidence rate ratio; BZD: Benzodiazepine or non-benzodiazepine derivatives (Z-drugs): Cl: Confidence interval; IRR: Incidence rate ratio; MMSE: Mini mental state examination; MRC CFAS: Medical research council cognitive function and ageing study

\section{Acknowledgments}

We would like to thank the MRC CFAS study group for data collection and management. We are also grateful to all respondents, their families and their primary care teams for their participation in the MRC CFAS. We would like to thank Mr. Barry Plumpton, Mrs. Ann McLauchlan, Mrs. Barbara di Vita, and Mrs. Gloria Swan for providing valuable assistance in interpretation and oversight as Alzheimer's Society Research Network Volunteers.

\section{Authors' contributions}

$\mathrm{KR}, \mathrm{GMS}, \mathrm{CF}, \mathrm{IM}, \mathrm{NS}, \mathrm{AA}, \mathrm{PKM}$, and YKL designed the study. CMG, GMS and $\mathrm{KR}$ designed the statistical analysis plan and CMG analysed the data. CMG, $\mathrm{KR}$, IM, CF, NC and MB assisted with coding the medication data. CB, FEM, $A A$, and LR organised the data collection. CMG and GMS wrote the initial draft of the paper, and CMG, GMS and KR amended the paper incorporating co-author comments. All authors read and approved the final manuscript.

\section{Funding}

This work was supported by the UK Alzheimer's Society [AS-PG-2013-017]. The funders had no role in the design of the study or the interpretation of the findings.

\section{Availability of data and materials}

Data can be shared through application. For further information please refer to the application forms on the website http://www.cfas.ac.uk/cfas-i/data/ \#cfasi-data-request

\section{Ethics approval and consent to participate}

Written consent was obtained for participation in the CFAS study. Ethical approval was obtained locally at all sites from 1991 and at Multi-centre research ethics committees during the course of the study (further detail on all ethical approvals can be found at http://www.cfas.ac.uk/files/2015/07/Ethical-approvals-for-CFAS.pdf). For example, the first multi-centre ethical approval was obtained at the Anglia \& Oxford multi-centre research ethics committee (ref: 99/5/22)

\section{Consent for publication}

Not applicable.

\section{Competing interests}

The authors declare that they have no competing interests, other than IM has received personal fees for guest lectures and to support travel from Astellas Pharmaceuticals; YL reports personal fees from Thame Pharmaceuticals, NC and CF have received grants and personal fees from Astellas Pharmaceuticals.

\section{Author details}

${ }^{1}$ School of Health Sciences, University of East Anglia, Norwich NR4 7TJ, UK. ${ }^{2}$ Norwich Medical School, University of East Anglia, Norwich NR4 7TJ, UK. ${ }^{3}$ School of Life and Health Sciences, Aston University, Birmingham B4 7ET, UK. ${ }^{4}$ Institute of Applied Health Sciences, University of Aberdeen, Aberdeen AB25 2ZD, UK. ${ }^{5}$ Department of Pharmacy Practice, College of Pharmacy, Purdue University, West Lafayette, USA. ${ }^{6}$ Indiana University School of Medicine, Indianapolis, Indiana, USA. ${ }^{7}$ Institute of Health and Society/Institute for Ageing, Newcastle University, Newcastle NE4 5PL, UK. ${ }^{8}$ Cambridge Institute of Public Health, University of Cambridge, Cambridge CB2 OSR, UK. ${ }^{9}$ Quadram Institute Bioscience, Norwich, Norfolk, UK.

Received: 7 February 2019 Accepted: 13 September 2019 Published online: 21 October 2019

\section{References}

1. Livingston G, Sommerlad A, Orgeta V, Costafreda SG, Huntley J, Ames D, et al. Dementia prevention, intervention, and care. Lancet Lond Engl. 2017; 390(10113):2673-734

2. Tannenbaum C, Paquette A, Hilmer S, Holroyd-Leduc J, Carnahan R. A systematic review of amnestic and non-amnestic mild cognitive impairment induced by anticholinergic, antihistamine. GABAergic Opioid Drugs Drugs Aging. 2012;29:639-58 
3. Olfson M, King M, Schoenbaum M. Benzodiazepine use in the United States. JAMA Psychiatry. 2015;72:136-42.

4. Sura SD, Carnahan RM, Chen H, Aparasu RR. Prevalence and determinants of anticholinergic medication use in elderly dementia patients. Drugs Aging. 2013;30:837-44.

5. National Institute for Health and Care Excellence. Urinary incontinence in women: management. NICE guideline CG171. 2015. https://www.nice.org. uk/guidance/cg171?unlid=. Accessed 10 Feb 2017.

6. Fick DM, Cooper JW, Wade WE, Waller JL, Maclean JR, Beers MH. Updating the Beers criteria for potentially inappropriate medication use in older adults: results of a US consensus panel of experts. Arch Intern Med. 2003; 163:2716-24.

7. Gray SL, Anderson ML, Dublin S, Hanlon JT, Hubbard R, Walker R, et al. Cumulative use of strong anticholinergic medications and incident dementia. JAMA Intern Med. 2015:175:401-7.

8. Carriere I, Fourrier-Reglat A, Dartigues J-F, Rouaud O, Pasquier F, Ritchie K, et al. Drugs with anticholinergic properties, cognitive decline, and dementia in an elderly general population: the 3-City study. Arch Intern Med. 2009;169:1317-24.

9. Fox C, Richardson K, Maidment ID, Savva GM, Matthews FE, Smithard D, et al. Anticholinergic medication use and cognitive impairment in the older population: the Medical Research Council cognitive function and ageing study. J Am Geriatr Soc. 2011;59:1477-83.

10. Chatterjee S, Bali V, Carnahan RM, Johnson ML, Chen H, Aparasu RR. Anticholinergic medication use and risk of dementia among elderly nursing home residents with depression. Am J Geriatr Psychiatry Off J Am Assoc Geriatr Psychiatry. 2016;24:485-95.

11. Boustani M, Campbell N, Munger S, Maidment I, Fox C. Impact of anticholinergics on the aging brain: a review and practical application. Aging Health. 2008:4:311-20.

12. Richardson K, Fox C, Maidment I, Steel N, Loke YK, Arthur A, et al. Anticholinergic drugs and risk of dementia: case-control study. BMJ. 2018; 361:k1315.

13. Rhee Taeho Greg, Choi Yookyung Christy, Ouellet Gregory M., Ross Joseph S. National Prescribing Trends for High-Risk Anticholinergic Medications in Older Adults. J Am Geriatr Soc. 0. https://doi.org/10.1111/jgs.15357.

14. Sumukadas D, McMurdo MET, Mangoni AA, Guthrie B. Temporal trends in anticholinergic medication prescription in older people: repeated crosssectional analysis of population prescribing data. Age Ageing. 2014;43:515-21.

15. Gray SL, Dublin S, Yu O, Walker R, Anderson M, Hubbard RA, et al. Benzodiazepine use and risk of incident dementia or cognitive decline: prospective population based study. BMJ. 2016:352:190.

16. Gomm W, von Holt K, Thomé F, Broich K, Maier W, Weckbecker K, et al. Regular benzodiazepine and Z-substance use and risk of dementia: an analysis of German claims data. J Alzheimers Dis JAD. 2016;54:801-8.

17. Billioti de Gage S, Begaud B, Bazin F, Verdoux H, Dartigues J-F, Peres K, et al. Benzodiazepine use and risk of dementia: prospective population based study. BMJ. 2012;345:e6231.

18. Billioti de Gage $S$, Moride $Y$, Ducruet $T$, Kurth $T$, Verdoux $H$, Tournier M, et al. Benzodiazepine use and risk of Alzheimer's disease: case-control study. BMJ. 2014;349:95205.

19. Imfeld P, Bodmer M, Jick SS, Meier CR. Benzodiazepine use and risk of developing Alzheimer's disease or vascular dementia: a case-control analysis. Drug Saf. 2015;38:909-19.

20. Wu C-S, Wang S-C, Chang I-S, Lin K-M. The association between dementia and long-term use of benzodiazepine in the elderly: nested case-control study using claims data. Am J Geriatr Psychiatry Off J Am Assoc Geriatr Psychiatry. 2009;17:614-20.

21. Wu C-S, Ting T-T, Wang S-C, Chang I-S, Lin K-M. Effect of benzodiazepine discontinuation on dementia risk. Am J Geriatr Psychiatry. 2011;19:151-9.

22. Richardson K, Mattishent K, Loke YK, Steel N, Fox C, Grossi CM, et al. History of benzodiazepine prescriptions and risk of dementia: possible Bias due to prevalent users and covariate measurement timing in a nested case-control study. Am J Epidemiol. https://doi.org/10.1093/aje/kwz073.

23. Brayne C, McCracken C, Matthews FE. Cohort profile: the Medical Research Council cognitive function and ageing study (CFAS). Int J Epidemiol. 2006:35:1140-5.

24. Copeland JRM, Dewey ME, Griffiths-Jones HM. Dementia and depression in elderly persons: Agecat compared with dsm III and pervasive illness. Int J Geriatr Psychiatry. 1990;5:47-51.
25. Matthews FE, Arthur A, Barnes LE, Bond J, Jagger C, Robinson L, et al. A two-decade comparison of prevalence of dementia in individuals aged 65 years and older from three geographical areas of England: results of the cognitive function and ageing study I and II. Lancet. 2013;382:1405-12.

26. Matthews F, Brayne C. The incidence of dementia in England and Wales: findings from the five identical sites of the MRC CFA study. PLoS Med. 2005;2:e193.

27. Richardson K, Kenny RA, Peklar J, Bennett K. Agreement between patient interview data on prescription medication use and pharmacy records in those aged older than 50 years varied by therapeutic group and reporting of indicated health conditions. J Clin Epidemiol. 2013.

28. Campbell NL, Maidment I, Fox C, Khan B, Boustani M. The 2012 update to the anticholinergic cognitive burden scale. J Am Geriatr Soc. 2013;61:S1-S232.

29. Melzer D. Socioeconomic status and the expectation of disability in old age: estimates for England. J Epidemiol Community Health. 2000;54:286-92.

30. Zou G. A modified Poisson regression approach to prospective studies with binary data. Am J Epidemiol. 2004;159:702-6.

31. Huerta C, Abbing-Karahagopian V, Requena G, Oliva B, Alvarez Y, Gardarsdottir H, et al. Exposure to benzodiazepines (anxiolytics, hypnotics and related drugs) in seven European electronic healthcare databases: a cross-national descriptive study from the PROTECT-EU project. Pharmacoepidemiol Drug Saf. 2016;25(Suppl 1):56-65.

32. Richardson K. Anticholinergic Medicine use and Dementia. BMJ. 2018;100:100.

33. Cai X, Campbell N, Khan B, Callahan C, Boustani M. Long-term anticholinergic use and the aging brain. Alzheimers Dement. 2012. https:// doi.org/10.1016/j.jalz.2012.02.005

34. Perry EK, Kilford L, Lees AJ, Burn DJ, Perry RH. Increased Alzheimer pathology in Parkinson's disease related to antimuscarinic drugs. Ann Neurol. 2003;54:235-8.

35. Whalley $\amalg$, Sharma S, Fox HC, Murray AD, Staff RT, Duthie AC, et al. Anticholinergic drugs in late life: adverse effects on cognition but not on Progress to dementia. J Alzheimers Dis. 2012;30:253-61.

36. Campbell NL, Boustani MA, Lane KA, Gao S, Hendrie H, Khan BA, et al. Use of anticholinergics and the risk of cognitive impairment in an African American population. Neurology. 2010;75:152-9.

37. Chuang Y-F, Elango P, Gonzalez CE, Thambisetty M. Midlife anticholinergic drug use, risk of Alzheimer's disease, and brain atrophy in community-dwelling older adults. Alzheimers Dement Transl Res Clin Interv. 2017;3:471-9.

38. Grymonpre R, Didur C, Montgomery P, Sitar D. Pill count, self-report, and pharmacy claims data to measure medication adherence in the elderly. Ann Pharmacother. 1998:32:749-54.

39. Connolly A, Gaehl E, Martin H, Morris J, Purandare N. Underdiagnosis of dementia in primary care: variations in the observed prevalence and comparisons to the expected prevalence. Aging Ment Health. 2011;15:978-84.

\section{Publisher's Note}

Springer Nature remains neutral with regard to jurisdictional claims in published maps and institutional affiliations.

Ready to submit your research? Choose BMC and benefit from:

- fast, convenient online submission

- thorough peer review by experienced researchers in your field

- rapid publication on acceptance

- support for research data, including large and complex data types

- gold Open Access which fosters wider collaboration and increased citations

- maximum visibility for your research: over $100 \mathrm{M}$ website views per year

At BMC, research is always in progress.

Learn more biomedcentral.com/submissions 